\title{
Aspects of Carbohydrate Metabolism.
}

\section{II.}

INVESTIGATION of the utilisation by the body of various compounds related to the sugars may be expected to throw light upon the intermediary metabolism of the carbohydrates and is, at any rate, of scientific interest. In this connexion, dihydroxyacetone, methylglyoxal, and glyceric aldehyde may be cited. Although the former antidotes insulin hypoglyeæmia in both man and animals, yet, administered by mouth to human beings on a fasting stomach, it itself produces a definite hypoglycæmia (E. P. Cathcart and J. Markowitz: Biochem. Jour, vol. 21, p. $1419 ; 1927)$. The fall may be compared with the secondary decrease in the blood-sugar after the ingestion of glucose, and may be due to stimulation of secretion of insulin from the pancreas. At the same time, the increased tension of carbohydrate in the liver may arrest the processes of glyconeogenesis and glycogenolysis.

In the rabbit, on the other hand, dihydroxyacetone raises the blood sugar (M. W. Goldblatt: ibid., vol. 22 , p. $464 ; 1928)$. In comparison with glucose this author found that dihydroxyacetone raised the respiratory quotient and increased the oxygen consumption to a greater extent but was not so effective in inhibiting the ketosis produced experimentally in man by starvation. Starvation reduces the sugar tolerance and the respiratory quotient is not raised so much as normally following the ingestion of carbohydrate. The antiketogenic influence of glucose is exerted simultaneously with the maximum increase in oxygen intake, that of dihydroxyacetone later. It appears that the latter forms glycogen less readily than glucose and cannot be considered as an intermediate stage in the breakdown of the glucose molecule. In muscle, on the other hand, W. O. Kermack, C. G. Lambie, and R. H. Slater failed to demonstrate any significant difference in the power of glucose or dihydroxyacetone to cause deposition of glycogen ( $i b i d$. vol. 23, p. $416 ; 1929)$. The experiments were carried out on decerebrate, depancreated cats, in which the liver remained in circulation.

Glyceric aldehyde is another compound which may play a part in the intermediary metabolism of carbohydrate. H. G. Reeves, however, found that it was toxic to the isolated heart of the rabbit, although it could augment and accelerate the beat of the frog's or toad's heart (Quart. Jour. Exp. Physiol., vol. 18, p. $277 ; 1927)$. It is possible that the difference between the mammalian and amphibian hearts is due to the difference in the temperatures at which the perfusions are necessarily carried out. It appears, therefore, that, at any rate in mammals, glyceric aldehyde is not an intermediate in the utilisation of glucose by cardiac muscle.

Goldblatt has found that carbohydrate metabolism is seriously interfered with in man by the administration of alkali, with the consequent production of a state of alkalosis (Biochem. Jour., vol. 21, p. 991 ; 1927). Glucose by mouth now increases the bloodsugar to a greater extent than normally and some is excreted in the urine; at the same time the rise in the respiratory quotient is less marked. These results indicate that both the storage and oxidation of carbohydrate are depressed. Similarly, alkali decreases the response of the rabbit to an injection of insulin, as well as the deposition of glycogen in the liver and muscles of the rat.

A considerable amount of work has been carried out on the carbohydrate metabolism of isolated tissues. G. S. Eadie has examined the conditions of action of rat's liver amylase (Biochem. Jour., vol. 21, p. 314;
1927). The enzyme was obtained by drying the organ with acetone and extracting the powder with 50 per cent glycerol, afterwards filtering and dialysing the extract. It was found that the liver enzyme was contaminated with blood amylase unless the organ was first perfused with Ringer's solution. The optimum $p H$ of liver amylase is at 6 , whilst that of blood amylase is at 6.8-6.9. The optimum salt concentration is 0.1 per cent; addition of adrenaline has no effect upon its activity. The enzyme and its substrate glycogen coexist in the same cell. E. F. Lesser (ibid., p. 1128) considers that the amylase is to a large extent adsorbed upon surfaces within the cell and so unable to act upon the glycogen.

J. T. Irving has investigated the glucose metabolism of kidney tissue in vitro (Biochem. Jour., vol. 22 , p. $964 ; 1928$ ). In the presence of oxygen, but not in its absence, chopped cortex incubated in phosphate or carbonate buffer converts glucose to lactic acid together with a small amount of hexose diphosphate; at the same time glycogen is utilised. The mechanism is therefore different from that found in muscle.

E. G. Holmes has continued his researches upon the metabolism of nervous tissue (Biochem. Jour., vol. 23, p. 1182 ; 1929 ; with C. A. Ashford, ibid., p. 748 ; with R. W. Gerard, ibid., p. 738 ; and with M. Sherif, ibid., vol. 24, p. $400 ; 1930$ ). In the case of brain it was found that free phosphate was liberated on both anaerobic and aerobic incubation, in the presence or in the absence of glucose ; no evidence of the synthesis of hexose phosphate in the process of formation of lactic acid was obtained, although the tissue can perform this operation to a small extent. Sodium fluoride inhibits this liberation of phosphate, when present in high concentration; even low concentrations markedly inhibit the formation of lactic acid, so that the two processes appear to be independent; in fact, it was found that lactic acid is freely formed from glucose in the absence of phosphate and its addition does not increase the velocity of formation of the acid. Brain tissue forms less lactic acid from glycogen than from glucose; sodium fluoride inhibits both phosphate and acid production, and removal of phosphate stops the formation of acid. It appears, therefore, that there are two lactic acid mechanisms in brain : one, quantitatively the more important, involves glucose and is independent of phosphate; the other involves glycogen and depends on the availability of phosphate. The source of the phosphate in the former case is obscure.

On anaerobic incubation, mammalian nerve produces lactic acid, two-thirds of which come from the free carbohydrate of the tissue and one-third from glycogen; in oxygen, no lactic acid is formed, but there is a definite fall in the free carbohydrate. Preformed lactic acid is not removed in oxygen (as is the case with brain and muscle). The oxygen consumption of nerve is much less than that of brain; about 60 per cent of the resting metabolism can be accounted for by the observed oxidation of carbo. hydrate. These carbohydrate changes apparently play no part in the extra metabolism of stimulation, since the nerve will still give a response after disappearance of all the carbohydrate. As the carbohydrate is consumed the oxygen consumption of resting nerve decreases ; in the presence of small amounts of glucose or galactose, however, the consumption remains linear for several hours ; brain, on the other hand, does not apparently use galactose.

The chemical composition and metabolism of non-

No. 3184, VoL. 126] 
medullated nerve are different from those of medullated; for these experiments Holmes used the nerves of crabs. The free carbohydrate, and especially the glycogen content, is very much higher; the latter may form 20 per cent of the total solids. Under anaerobic conditions at rest, the glycogen decreases, with a coincident increase in the free carbohydrate and lactic acid; in oxygen the fall in glycogen and the rise in free sugar are considerably less, and there is no increase in lactic acid, although preformed lactic acid is not removed. In the nerve-ganglia the presence of a polysaccharide was detected.

J. Pryde and R. W. Humphreys (Biochem. Jour., vol. 20 , p. 825 ; 1926) have shown that the oxidic bridge of the galactose in the cerebrosides of ox brain is of the stable amylene type.

The carbohydrate metabolism of cancer cells differs from that of most normal tissues in that aerobic glycolysis is a prominent feature, whilst the oxidative removal of lactic acid is a relatively slow process. B. E. Holmes (Biochem. Jour., vol. 20, p. 812; 1926) has shown that certain tumour tissues contain very little reduced glutathione and have only a slight activity in reducing the oxidised form when added. H. G. Crabtree (ibid., vol. 22, p. $1289 ; 1928$ ) has found that certain pathological overgrowths, aroused by different viruses, behave like malignant tissue in their carbohydrate metabolism; this change from the normal was not seen when the virus failed to produce hyperplasia.

F. Dickens and F. Simer (Lancet, vol. 2, p. 10; 1930) have recently shown that the respiratory quotient of normal tissues runs parallel with their power of anaerobic glycolysis; tumours have a low respiratory quotient, indicating a poor ability to oxidise carbohydrate, although actively glycolytic. The inability to oxidise lactic acid is peculiar, since tumours, like normal tissues, oxidise pyruvic acid.

S. T. Harrison and E. Mellanby (Biochem. Jour., vol. 24, p. $141 ; 1930$ ) have investigated the inhibitory action of pancreatic and other extracts upon the formation of lactic acid in cancer and muscle. It has been known for some time that pancreatic extracts inhibit acid production by muscle hash. D. $R$. McCullagh (ibid., vol. 22, p. 402 ; 1928) confirmed this for muscle extract; he also showed that the former prevented the disappearance of carbohydrate, but caused an increase in the free phosphate content instead of a decrease. In the presence of sodium fluoride, pancreatic extract prevented the formation of hexose phosphate, and the author considers that this is the cause of the failure to produce lactic acid. In a later paper, working with E. M. Case, it was found that the inhibition was probably due to the amylase present in the pancreatic extract (ibid., p. 1060).

The properties of the unknown factor and the enzyme are very similar: inhibition is observed when malt or taka diastase replaces the pancreatic extract, or when glycogen is used as substrate instead of starch; and the formation of lactic acid from activated glucose by muscle extract is not inhibited. Harrison and Mellanby confirmed the inhibition when starch is used as the source of the lactic acid, and the failure of inhibition with glucose ; they also found, however, that inhibition occurs with hexose diphosphate but not with hexose monophosphate. They therefore consider that the pancreatic extract does not act by inhibiting the esterification of hexose but by forming maltose, which is only slightly acted upon by the muscle system with the production of lactic acid. They agree that the inhibition is due to amylase; the inhibition of lactic acid formation from hexose diphosphate, however, cannot yet be explained. The same authors have also shown that various preparations of amylase inhibit the glycolysis, anaerobic and aerobic, of tumour tissue; the latter cannot form lactic acid from hexose di- or mono-phosphate or from starch to any appreciable extent, so that in this case also the inhibition cannot be due to inhibition of ester formation.

Pancreatic extracts have also been reported to contain an antiglyoxalase. Phenylglyoxal is converted to mandelic acid by liver extract; pancreatic extract inhibits the reaction. J. O. Giršavičius (Biochem. Jour., vol. 24, p. 446 ; 1930) has found that pancreatic extract itself produces acid from phenylglyoxal; a reaction appears to occur between the phenylglyoxal and diamino-acids and polypeptides in the extract with production of an orange substance. It does not appear that this reaction can be responsible for the antiglyoxalase activity of pancreatic extracts, since the substances involved are thermostable and dialysable.

\section{Mosses as Epiphytes.*}

$\mathrm{W}$ ISNIEWSKI has surveyed parts of the virgin forest of Białowieza, with special reference to the epiphytic Bryophyta. His results and the discussion of their significance bring out several points of interest bearing on the nature of epiphytism in this group. He recognises four associations of mosses on the trees, the first two of which appear to be true epiphytes, whilst the other two are more closely related to the vegetation of the ground flora.

The two epiphytic associations are :

(1) On broad-leaved trees (except Betula spp.) : Leucodon sciuroides (chiefly on Carpinus betulus) and Anomodon viticulosus (chiefly on Acer platanoides and Fraxinus excelsior).

(2) On coniferous trees and Betula spp.: Drepanium (Hypnum) cupressiforme, var. filiforme and Orthodicranum montanum.

The other two associations under consideration are: (3) In damper situations on any type of tree : Eurhynchium striatum.

* Wisniewski, T. “ Les associations des Muscinées (Bryophyta) épiphytes de la Pologne, en particulier celles de la forêt vierge de Białowieza." (Bull. Internat. de l'Acad. Polonaise des Sci. et des Lettres, pp. 293-342 ; 1929.)

No. 3184, VoL. 126]
(4) In drier situations on any type of tree : Pleurozium Schreberi.

Considering possible factors influencing the ability of mosses to grow in different situations, it is clear that neither light nor temperature is a limiting factor. The light intensity lies well within the limits at which at least some of the species under consideration have been found to flourish, according to the work of Zmuda and Malta, and the same applies to the temperature range. The question of water supply is, however, a vital one for mosses as their method of water absorption is entirely different from that of root-bearing plants. It is clear from the work of Schimper and others that the rhizoids of a moss are ineffective in the uptake of water, for even if the lower parts of a moss plant are actually in water, the upper parts may be seen to wilt. The chief method of water absorption seems to be imbibition by the walls over the whole surface of the plant, whether dead or living, as is illustrated by the rapid recovery of form on moistening dry specimens. Wisniewski found an interesting difference in this respect between the epiphytic mosses and those of the ground flora, for whilst the dry plants of the former group 